\title{
Evaluating the Efficacy of Microplates along with Platelet-Rich Fibrin in Osteosynthesis of Mandibular Angle Fracture Management
}

\author{
Guntoju Swetha, B. Pavan Kumar, V. Vidya Devi \\ Department of Oral and Maxillofacial Surgery, Kamineni Institute of Dental Sciences, Nalgonda, Telangana, India
}

Email for correspondence: swetha.swethu127@gmail.com

\begin{abstract}
Introduction: The treatment of mandibular fractures has been in a constant state of evolution with goals to restore function and deranged occlusion with proper fixation techniques until stable osseointegration is achieved. Aim: The present study was conducted to evaluate and assess the clinical and functional stability of the fracture fragments that are fixed with microplates and screws and to assess the efficacy of the platelet-rich fibrin (PRF) when placed between the fracture fragments. Materials and Methods: The present study was conducted in the Department of Oral and Maxillofacial Surgery, Kamineni Institute of Dental Sciences, Narketpally, Nalgonda, Telangana. A total of 30 patients ( 24 males and 6 females) of age 16-70 years were selected for the study with mandibular angle fracture which is fixed with microplates and screws, PRF was placed between the fracture fragments. Bite force is measured using bite force transducer and bone healing was measured using the orthopantomogram, pain is measured using visual analog scale, and all the findings were recordings which were statistically analyzed. Results: Paired " $t$-test" and Kruskal-Wallis test showed that there was statistically significant difference between the pre-operative and post-operative bone healing and bite forces. The mean bite forces preoperatively for incisors, right molar, and left molar are $3.4 \pm 0.95,8.9 \pm 5.9$, and $12.79 \pm 6.4$, respectively, and post-operative bite forces at 1 week are $3.57 \pm 0.96$, $13.02 \pm 6.3$, and $9.4 \pm 6.07$, at 1 month they are $4.15 \pm 0.89,14.8 \pm 5.8$, and $12.4 \pm 4.9$, and at 3 months they are 4.76 $\pm 0.5573,17.4 \pm 4.1$, and $17.01 \pm 4.005$, respectively. The mean normal bone density that is measured is $164.8 \pm 11.7$ and post-operative bone density at immediate post-operative, 1 month, and 3 months is $118.3 \pm 10.18,134 \pm 7.07$, and $143.4 \pm 9.23$, respectively. Conclusion: Microplates along with PRF can be used in the osteosynthesis.
\end{abstract}

Key words: Mandibular angle fracture management, microplates, osteosynthesis, platelet-rich fibrin

\section{INTRODUCTION}

Rapid advancement in the quality of life, road traffic accidents, assaults, interpersonal violence, sports injuries, falls, etc., have alarmingly increased and are the cause of serious concern to clinician and health-care service providers. Miniplate osteosynthesis, introduced by Michelet et al., in 1973, and further developed by Champy et al., in 1978,

\begin{tabular}{ll}
\hline Quick Response Code & Article Info: \\
\hline doi: 10.5866/2019.11.10093 & $\begin{array}{l}\text { Received: 08-07-2019 } \\
\text { Revised: 09-08-2019 } \\
\text { Accepted: 17-08-2019 } \\
\text { Available Online: 01-10-2019, (www. } \\
\text { nacd.in) @ NAD, 2019 - All rights reserved }\end{array}$ \\
\hline
\end{tabular}

has become the standard treatment of mandibular fractures. ${ }^{[1]}$ Miniplates and reconstruction plates are commonly used to treat simple and comminuted fractures. However, thick fixation plates are bulky and palpable through the skin and the gingiva. There is limited space available in the upper half of the mandible for a large miniplate, which can result in complications such as infection, wound healing problems, tooth root injuries, or mass effect problems. ${ }^{[2]}$

Previously, microplates were used in nonstress-bearing areas such as mid-face, but recent experiments and clinical studies have shown that microplates can be used efficiently in stressbearing areas such as mandible. There are many studies which have shown the effect of trauma on 
masticatory and biting forces. In a traumatized mandible, the environment of soft and hard tissue, both are affected. Thus, masticatory and biting forces are altered and in fact significantly reduced in the new environment. ${ }^{[3]}$ In this specific situation, the use of $2.0 \mathrm{~mm}$ standard miniplates appears to be big and bulky and the use of $1.2 \mathrm{~mm}$ microplates may be a reasonable choice as tensile strength of titanium is more than the tensile strength of bone and mandible is significantly low. Hence, microplates can be used in place of miniplates as they are smaller and lighter than them.

Platelet-rich fibrin (PRF) is an immune and platelet concentrate collecting on a single fibrin membrane all the constituents of a blood sample favorable to healing and immunity. ${ }^{[4]}$

The angiogenesis property of fibrin matrix is explained by the three-dimensional (3D) structure of the fibrin gel and by the simultaneous action of cytokines trapped in the meshes. Furthermore, main angiogenesis soluble growth factors such as fibroblast growth factor basic, vascular endothelial growth factor (VEGF), angiopoietin, and plateletderived growth factor (PDGF) are included in fibrin gel. These are the factors which promote soft and hard tissue healing. ${ }^{[5]}$

The present study was carried out in the department of oral and maxillofacial surgery, which has sophisticated equipment and infrastructure that are required to treat the maxillofacial trauma and evaluate the efficacy of microplate and with $\mathrm{PRF}$ in the angle fracture in osteosynthesis of mandibular angle fracture management.

\section{MATERIALS AND METHODS}

\section{Samples and Study Design}

The study consists of 30 patients with maxillofacial trauma which were drawn from the Kamineni Institute of Dental Sciences and Hospital and Outpatient Department of Oral and Maxillofacial Surgery, Sreepuram, Narketpally, Nalgonda, Telangana, over a period between January 2018 and July 2019 who need the mandibular angle fracture management.

\section{Study Protocol}

All the patients should undergo pre-surgical evaluation before treatment they are informed about the risks and complications of the procedure, informed consent was obtained, pre-operative clinical [Figure 1] and radiographical examination
[Figure 2] was done using orthopantomogram (OPG). Patients diagnosed to have mandibular angle fractures and need fixation, who also fulfill all the inclusion criteria are considered. Arch bar fixation was done preoperatively for every patient under local anesthesia [Figure 3]. General anesthesia is administered through oral/nasotracheal/submental depending on the case and local anesthesia is administered locally. The fracture site is exposed through intraoral approach [Figure 4] and, before the reduction of fracture fragments, the sufficient amount of venous blood is withdrawn and centrifuged for $10 \mathrm{~min}$ with $3000 \mathrm{rpm}$ and PRF that is obtained [Figure 5] is kept in between the fracture fragments followed by fixation with $1.5 \mathrm{~mm}$ microplates and screws [Figure 6]. Post-operative follow-up was

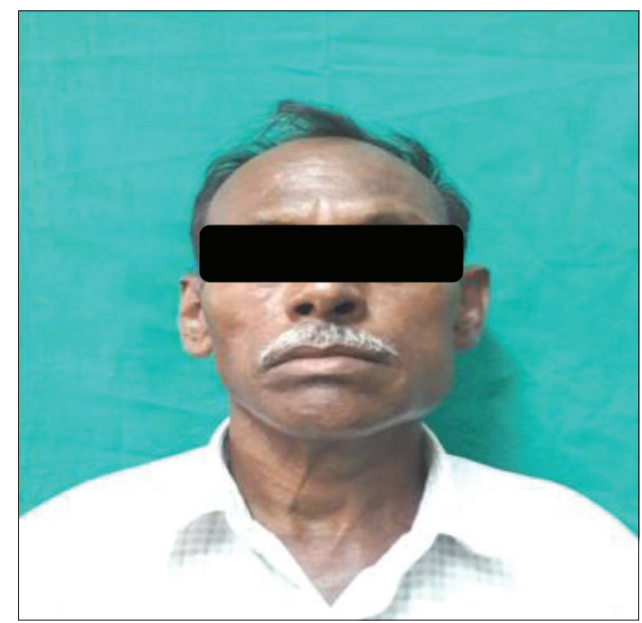

Figure 1: Frontal profile showing asymmetry of face on the left side

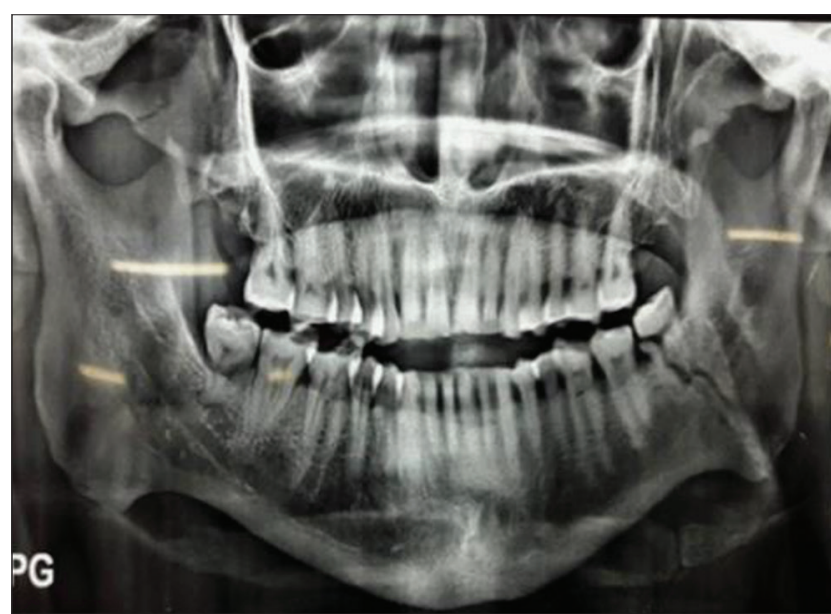

Figure 2: Pre-operative radiograph showing the left angle fracture 
done for 3 months and radiographs were taken to evaluate the progress of bone healing at immediate post-operative day [Figure 7], 1 month, and 3 months, respectively [Figure 8].

Bite forces are measured using a strain gauge bite force transducer made of stainless steel biting sensor of width $5 \mathrm{~mm} 10 \mathrm{~mm}$ and a microcontroller-based digital load indicator at preoperatively and postoperatively 1 week, 1 month, and 3 months, respectively. Bone healing was measure using the OPG immediate post-operative day, 1 month, and 3 months, respectively. Pain is assessed using a visual analog scale on preoperatively and postoperatively.

\section{RESULTS}

All the patients were treated with open reduction and internal fixation (ORIF) using

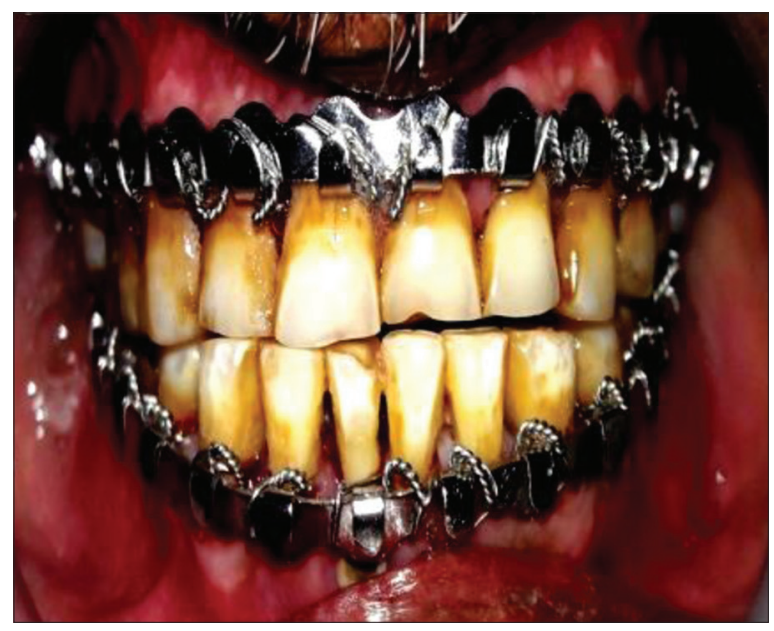

Figure 3: Pre-operative occlusion after arch bar fixation

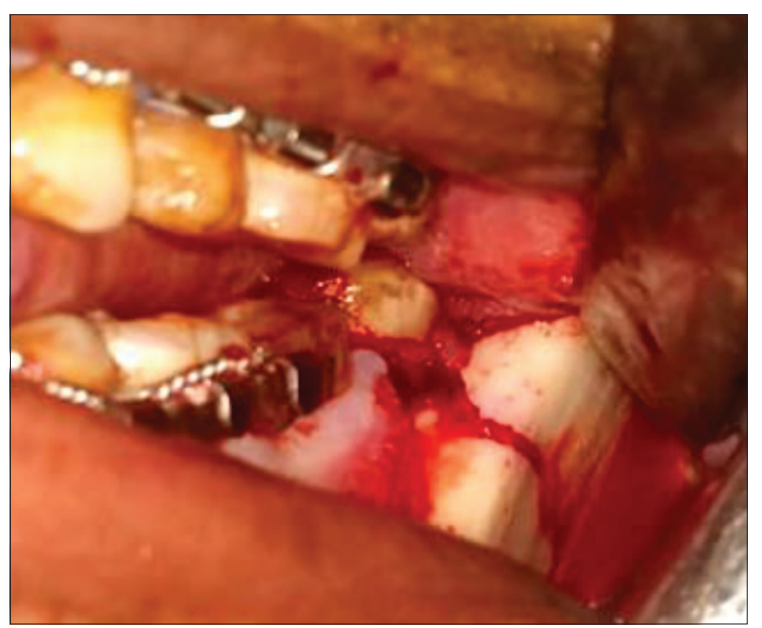

Figure 4: Intraoral incision given and fracture site exposed at the left angle region microplates and screws under general anesthesia, along with the placement of PRF in between the fractured segments to evaluate bone healing at the $1^{\text {st }}$ post-operative day, 1 month, and 3 months, respectively. Bite forces are measured preoperatively, postoperatively on week, 1 month, and 3 months, respectively, and pain is measured preoperatively and postoperatively on $3^{\text {rd }}$ day, $15^{\text {th }}$ day, and 1 month, respectively. All the data were collected and analyzed using the Statistical Package for the Social Sciences statistical analysis software (2019), Kruskal-Wallis and paired $t$-test.

Bone density was assessed postoperatively at $1^{\text {st }}$ post-operative day, 1 month, and 3 months in an OPG. There is the gradual increase in the

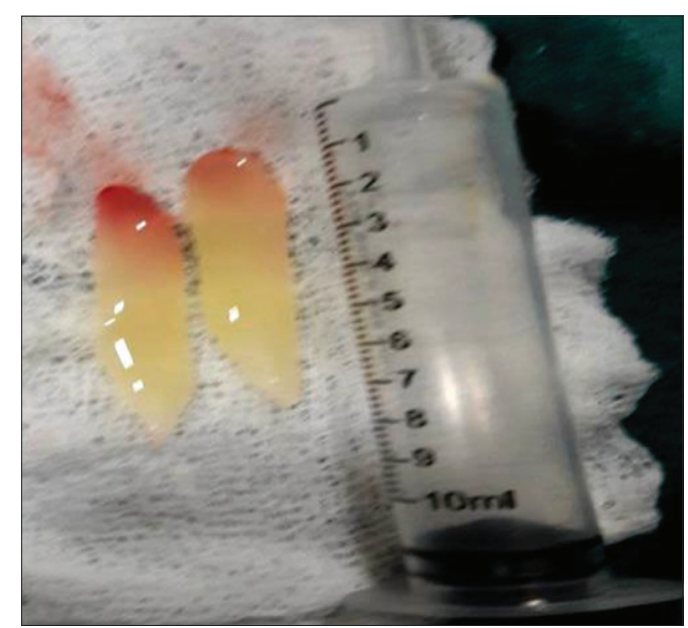

Figure 5: Length of the PRF obtained after centrifugation for $3000 \mathrm{rpm}$ for $9 \mathrm{~min}$

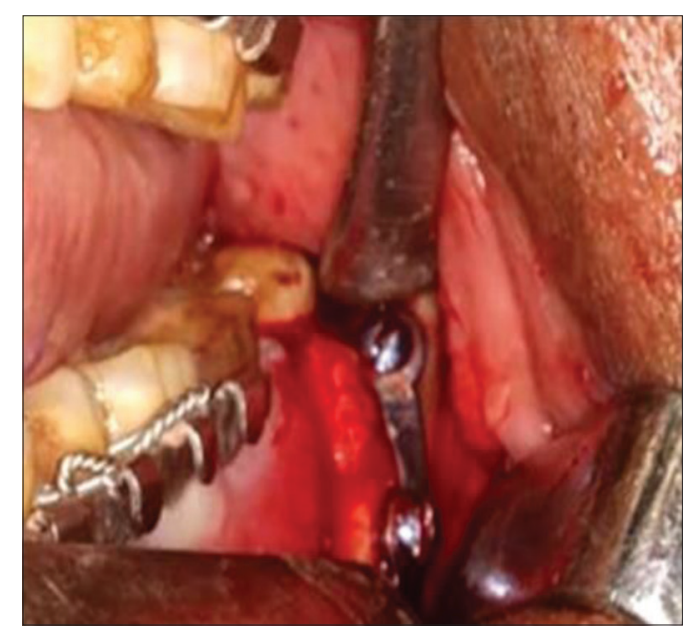

Figure 6: PRF placed in between the fracture fragments and fixed with microplates and screws 




Figure 7: Immediate post-operative radiograph showing the fixation of fracture with microplate and screws

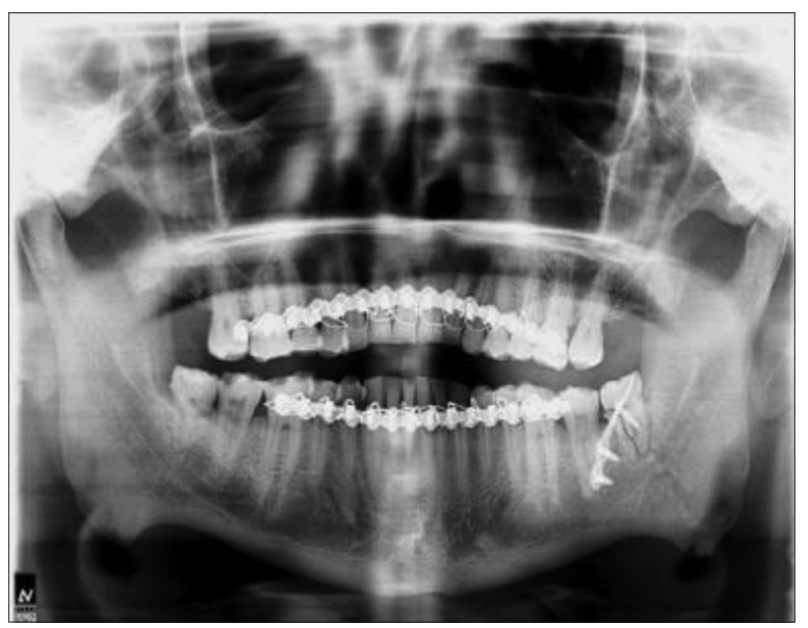

Figure 8: Post-operative radiograph after 3 months showing bone healing

bone density from the immediate post-operative day to 3 months post-operative day. The 3 -month post-operative bone density is near approximate to the normal bone density, which is statistically significant with $P<0.005$ [Graph 1].

Bite forces were recorded preoperatively and postoperatively on the $1^{\text {st }}$ post-operative day, 1 week, 1 month, and 3 months. The average bite forces of incisors, right molar, and left molar were recorded and were compared with pre-operative bite forces and found that the value of bite forces significantly increased $(P<0.001)$ both in incisor and molar region on both the sides at post-operative follow-up [Graph 2].

Pain was recorded in all patients postoperatively on the $1^{\text {st }}$ post-operative day, 1 week, 1 month,

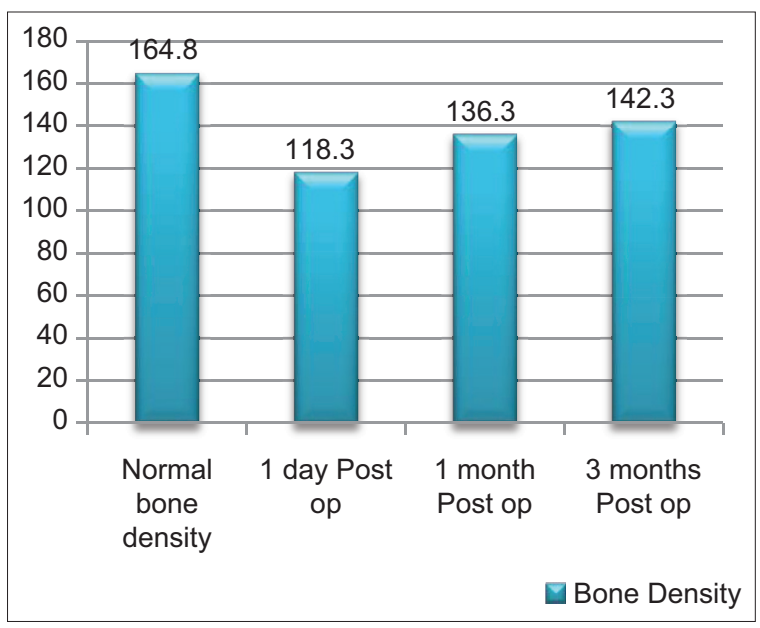

Graph 1: Comparing the normal bone density with post-operative bone density at immediate post-operative day, 1 month, and 3 months

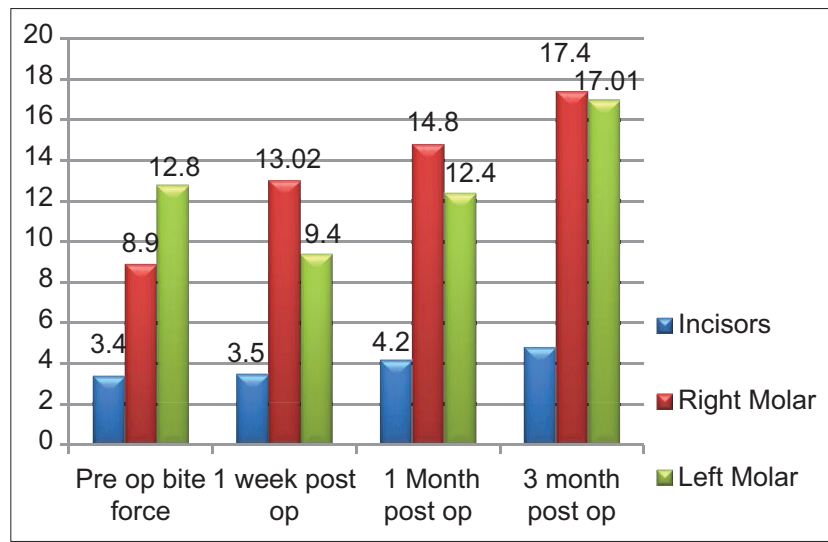

Graph 2: Graph showing the comparison of pre-operative and post-operative 1 week, 1 month, and 3 months

and 3 months, respectively. Pain was severe in all patients at the $3^{\text {rd }}$ post-operative day and gradually reduced to moderate pain at the $15^{\text {th }}$ post-operative day in all patients. Post-operative pain scores are compared; there is a significant $(P<0.005)$ decrease in the pain score from the $3^{\text {rd }}$ day post-operative day to 1 month postoperatively [Graph 3].

\section{DISCUSSION}

The goals of the mandibular fracture fixation are restoration of normal structure and function. Elements of successful repair include immobilization at the fracture site, habitual dental occlusion, and solid bony union, absences of infection, normal nerve function, and normal range of motion. ${ }^{[6]}$ The percentage of mandibular fracture site according to site is given as follows: Parasymphysis fracture 


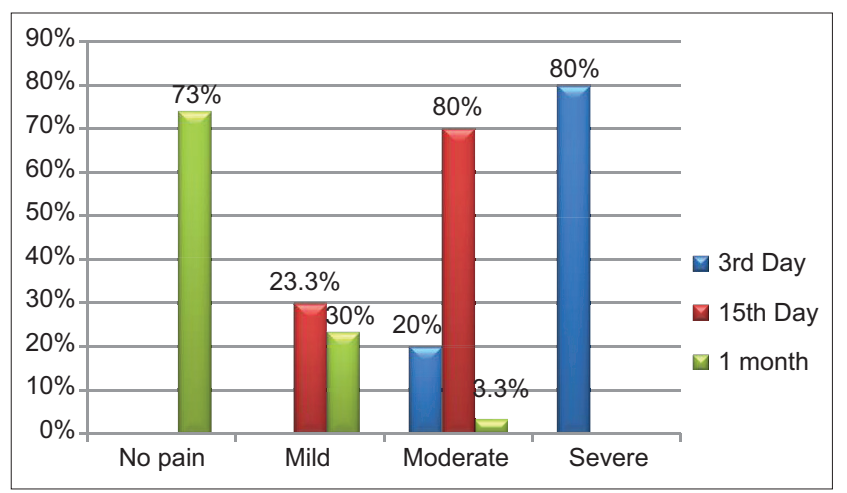

Graph 3: Graph showing the post-operative pain at the $3^{\text {rd }}$ day, $15^{\text {th }}$ day, and 1 month

(31.4\%), body (24.5\%), subcondyle (20.6\%), and angle $(13.7 \%)$ were the most common sites while fracture of condyle (1\%), coronoid (1.0\%), dentoalveolar (1.0\%), and ramus (1.0\%) was the least common fracture sites. ${ }^{[7]}$

A microfixation system (Luhr, 1987) was developed with extremely tiny plates and screws to achieve adequate $3 \mathrm{D}$ rigidity and at the same time with minimal interference with the overlying soft tissues. ${ }^{[8]}$ Previously, microplates were used in non-stress-bearing areas such as the mid-face, but recent experimental and clinical studies have shown that microplates can be used efficiently in stressbearing areas such as the mandible. Hegtvedt et al. (1994) compared the resistance of Luhr minisystem and Luhr microsystem in a biomechanical model by applying various in vitro forces such as edgewise bending, flat bending, tension, and compression and concluded that Luhr minisystem provides a significant amount of resistance to tensile and compressive forces. ${ }^{[8,9]}$

In the previous studies, there has been a lot of debate over one-point fixation versus two-point fixation in mandibular angle fractures. However, it has been demonstrated that as a result of neuromuscular protection, the masticatory loading forces are reduced to $30 \%$ in the first 6 weeks after fracture of the mandibular angle, increasing only slowly with time thereafter. ${ }^{[3]}$ In addition, it has been shown that sufficient stabilization of isolated, single mandibular angle fractures can be achieved by fixation with just one microplate along the external oblique ridge. ${ }^{[10]}$ Thus, it may be possible to maintain the stability of the fracture site using a less rigid fixation system that has been previously thought necessary.

\section{Advantages of Use of Microplates and Screws}

Microplates are now commonly used to restore maxillofacial fractures because:

- They require less manipulation

- They are more malleable and easier to adapt to the shape of the bone

- They are less likely to cause iatrogenic damage, and

- They are associated with a lower rate of major complications than miniplates ${ }^{[10]}$

For mandibular fractures, however, most surgeons have not established whether the microplate fixation system is strong enough to withstand masticatory forces until a stable bony union is achieved. Nevertheless, some clinical trials have also demonstrated that the microplates are strong enough to keep mandible fractures reduced. ${ }^{[10-12]}$

Schortinghuis et al. studied the complications associated with the internal fixation of maxillofacial fractures with microplates and found no hardware exposure or infection. ${ }^{[11]}$

Disadvantage of the microplate is that the plate, as well as the screws, is as follows:

- They are expensive

- They are chances of screw or drill breakage due to smaller diameter

- They may not have enough holding power, which leads to screw and plate loosening and secondary dislocation into the bone parts and leading to non-anatomic healing.

\section{PRF}

PRF has come up as the second-generation platelet concentrate with cicatricial properties. Its production protocol attempts to accumulate platelets and release cytokines in a fibrin clotdeveloped in France by Choukroun et al., since 2000. ${ }^{[13,14]}$

$\mathrm{PRF}$ is an immune and platelet concentrate collecting on a single fibrin membrane, containing all the constituents of blood favorable for healing and immunity. Through platelet and leukocyte, cytokines play an important part in the biology of this biomaterial; the fibrin matrix supporting them certainly constitutes the determining element responsible for the real therapeutic potential of PRF.

Angiogenesis, immunity, and epithelial cover are the three keys to healing and soft-tissue maturation. PRF is able to simultaneously support 
the development of these three phenomena. The angiogenesis property of fibrin matrix is explained by the $3 \mathrm{D}$ structures of the fibrin gel and by the simultaneous action of cytokines trapped in the meshes. ${ }^{[15]}$

The biologic properties of autologous platelet concentrates exploit the potential of several platelet growth factors (PDGF, transforming growth factor [TGF]-beta, EGF, VEGF, insulin-like growth factor [IGF-I], basic fibroblast growth factor [b-FGF], and hepatocyte growth factor) in them, PRF releases high quantities of three main growth factors TGF beta-1, PDGF-AB, VEGF, and an important coagulation matricellular glycoprotein (thrombospondin-1) during 7 days. Apart from these, PRF also secretes EGF, FGF, and three important pro-inflammatory cytokines - interleukin (IL)-1b, IL-6, and tumor necrosis factor- obtained with a simple centrifugation procedure, to stimulate several biological functions such as chemotaxis, angiogenesis, proliferation, differentiation, and modulation, thereby representing a possible therapeutic modality for a more rapid and effective regeneration of hard and soft tissues. ${ }^{[15-19]}$

\section{Maximum Voluntary Bite Force}

Maximum biting force is the greatest force that an individual can generate by voluntary clenching of teeth in the occlusal position. The measurement of forces in the oral cavity has evolved from the use of crude methods of placing measuring devices between the teeth, to the greater sophistication of strain gauges with leads to the recording equipment. Strain gauges were used by Howell and Manly, Fløystrand et al., and Bakke et al. for measuring bite forces. ${ }^{[20-22]}$

The measurement of bite forces has been remained a matter of interest among many researchers. However, there is inconsistency in the findings and maximum value of bite forces presented by different authors. ${ }^{[23,24]}$

According to Srikanth et al., 2018, the average maximum voluntary bite force recorded in the healthy individuals is in females: Incisors: $9.51 \pm 4.28 \mathrm{~kg}$, in the right and left first molars, it is $34.87 \pm 7.31 \mathrm{~kg}$ and $35.94 \pm 7.86 \mathrm{~kg} \cdot{ }^{[25]}$

The maximum voluntary bite forces in males in the present study are as follows: Incisors: $17.62 \pm 5.13 \mathrm{~kg}$ and in the right and left first molars, it is $46.38 \pm 13.33 \mathrm{~kg}$ and $49.10 \pm 15.16 \mathrm{~kg}$.
It has been established that biting and occlusive forces are reduced in traumatized mandible. ${ }^{[26-28]}$ A time-based assessment in the post-treatment phase of biting and occlusive forces would present a real picture of masticatory function which is done by concentrating first on the bite forces at different intervals after ORIF to evaluate the efficacy of microplate in mandibular angle fractures management.

\section{Bone Density}

In the present study, bone density was assessed postoperatively on the $1^{\text {st }}$ post-operative day and 1 month and $3^{\text {rd }}$ month after surgery on an OPG. There was the gradual increase in the bone density from the immediate post-operative day to the $3^{\text {rd }}$ post-operative month. The 3 -month postoperative bone density was close to the normal bone density, which was statistically significant with $P<0.005$. Our study is in accordance with other studies. A study was done by Al Rayess et al. (2018) on clinical and radiographic healing in mandibular fractures using PRF membrane with miniplate fixation and found that a PRF application on the fracture line in the mandible may be helpful in improvement of the fracture healing when compared to the control group though the difference was statistically significant. ${ }^{[29]}$

Another study by Zhang et al., 2012, in the study evaluated that influence of PRF on bone regeneration in sinus augmentation in combination with xenograft deproteinized bovine bone. ${ }^{[30]}$ The percentage of new bone formation in the PRF group was about 1.4 times of that in control, while the percentage of residual bone substitute in the control group was about 1.5 times higher as that in the PRF group. The percentage of contact length between newly formed bone and bone substitute in the PRF group was higher than the control group.

\section{CONCLUSION}

On the basis of our clinical findings, it can be concluded that microplate can be used for the management of angle fracture due to the added advantages such as smaller diameter of the plates and screws, higher corrosion resistance, light weightiness, and lesser toxicity, there is lesser chance of iatrogenic damages. As the restoration of masticatory function is the major prerequisite of any fracture fixation, assessment of bite forces can be pivotal for early mobilization and PRF placed in between the fracture segments promotes early bone 
regeneration, soft-tissue healing due to the presence of growth factors such as PDGF, TGF-1, and VEGF in the presence of thrombocytes.

\section{REFERENCES}

1. Michelet FX, Deymes J, Dessus B. Osteosynthesis with miniaturized screwed plates in maxillo-facial surgery. J Maxillofac Surg 1973;1:79-84.

2. Burm JS, Hansen JE. The use of microplates for internal fixation of mandibular fractures. Plast Reconstr Surg 2010;125:1485-92.

3. Tate GS, Ellis E $3^{\text {rd }}$, Throckmorton G. Bite forces in patients treated for mandibular angle fractures: Implications for fixation recommendations. J Oral Maxillofac Surg 1994;52:734-6.

4. Dohan DM, Choukroun J, Diss A, Dohan SL, Dohan AJ, Mouhyi J, et al. Platelet-rich fibrin (PRF): A secondgeneration platelet concentrate. Part I: Technological concepts and evolution. Oral Surg Oral Med Oral Pathol Oral Radiol Endod 2006;101:e37-44.

5. Sahni A, Odrljin T, Francis CW. Binding of basic fibroblast growth factor to fibrinogen and fibrin. J Biol Chem 1998:273:7554-9

6. Ghodke MH, Bhoyar SC, Shah SV. Prevalence of mandibular fractures reported at C.S.M.S.S dental college, aurangabad from february 2008 to september 2009. J Int Soc Prev Community Dent 2013;3:51-8.

7. Natu SS, Pradhan H, Gupta H, Alam S, Gupta S, Pradhan R, et al. An epidemiological study on pattern and incidence of mandibular fractures. Plast Surg Int 2012;2012:834364.

8. Luhr HG. A micro-system for cranio-maxillofacial skeletal fixation. Preliminary report. J Craniomaxillofac Surg 1988;16:312-4

9. Hegtvedt AK, Michaels GC, Beals DW. Comparison of the resistance of miniplates and microplates to various in vitro forces. J Oral Maxillofac Surg 1994;52:251-7.

10. Haug RH, Morgan JP $3^{\text {rd }}$. A microplate and screw technique for intraoral open reduction of mandibular angle fractures. J Oral Maxillofac Surg 1995;53:218-9.

11. Schortinghuis J, Bos RR, Vissink A. Complications of internal fixation of maxillofacial fractures with microplates. J Oral Maxillofac Surg 1999;57:130-4.

12. Eppley BL, Sadove AM. Application of microfixation techniques in reconstructive maxillofacial surgery. J Oral Maxillofac Surg 1991;49:683-8.

13. Marx RE, Carlson ER, Eichstaedt RM, Schimmele SR Strauss JE, Georgeff KR. Platelet-rich plasma: Growth factor enhancement for bone grafts. Oral Surg Oral Med Oral Pathol Oral Radiol Endod 1998;85:638-46.

14. Brown LF, Lanir N, McDonagh J, Tognazzi K, Dvorak AM, Dvorak HF. Fibroblast migration in fibrin gel matrices. Am J Pathol 1993;142:273-83.

15. Choukroun J, Adda F, Schoeffler C, Vervelle AP. Une opportunité en paro-implantologie: Le PRF. Implantodontie 2001;42:62

16. Kumar N, Prasad K, Ramanujam L, Ranganath K, Dexith J, Chauhan A. Evaluation of treatment outcome after impacted mandibular third molar surgery with the use of autologous platelet-rich fibrin: A randomized controlled clinical study. J Oral Maxillofac Surg 2015;73:1042-9.

17. Vivek GK, Sripathi Rao BH. Potential for osseous regeneration of platelet rich plasma: A comparitive study in mandibular third molar sockets. J Maxillofac Oral Surg 2009;8:308-11.

18. Giannobile WV. Periodontal tissue engineering by growth factors. Bone 1996;19:23S-37.

19. Intini G. The use of platelet-rich plasma in bone reconstruction therapy. Biomaterials 2009;30:4956-66.

20. Meyer RA. Nerve damage from fixation screws. J Ora Maxillofac Surg 1990;48:665

21. Howell AH, Manly RS. An electronic strain gauge for measuring oral forces. J Dent Res 1948;27:705-12.

22. Fløystrand F, Kleven E, Oilo G. A novel miniature bite force recorder and its clinical application. Acta Odontol Scand 1982;40:209-14.

23. Bakke M, Holm B, Jensen BL, Michler L, Möller E. Unilateral, isometric bite force in 8-68-year-old women and men related to occlusal factors. Scand J Dent Res 1990;98:149-58

24. Kshirsagar R, Jaggi N, Halli R. Bite force measurement in mandibular parasymphyseal fractures: A preliminary clinical study. Craniomaxillofac Trauma Reconstr 2011;4:241-4.

25. Srikanth MM, Kumar BP, Venkatesh V, Devi VV. Evaluation of bite forces in healthy individuals. Indian J Dent Adv 2018;10:101-5

26. Korkmaz HH. Evaluation of different miniplates in fixation of fractured human mandible with the finite element method. Oral Surg Oral Med Oral Pathol Oral Radiol Endod 2007;103:e1-13.

27. Koc D, Dogan A, Bek B. Bite force and influential factors on bite force measurements: A literature review. Eur J Dent 2010;4:223-32

28. Koç D, Do an A, Bek B. Effect of gender, facial dimensions, body mass index and type of functional occlusion on bite force. J Appl Oral Sci 2011;19:274-9.

29. Al Rayess AM, El Dibany MM, Melek LN. Clinical and radiographic study of healing of mandibular fractures using plasma rich fibrin membrane with miniplate fixation. Alex Dent J 2018;43:41-7.

30. Zhang Y, Tangl S, Huber CD, Lin Y, Qiu L, RauschFan X. Effects of choukroun's platelet-rich fibrin on bone regeneration in combination with deproteinized bovine bone mineral in maxillary sinus augmentation: A histological and histomorphometric study. J Craniomaxillofac Surg 2012;40:321-8 